\title{
Supporting Information \\ 2,2'-Dipyridylamine as Heterogeneous Organic Molecular Electrocatalyst for Two-Electron Oxygen Reduction Reaction in Acid Media
}

\author{
Xi Yin, Ling Lin, Ulises Martinez, Piotr Zelenay* \\ Materials Physics and Application Division, Los Alamos National Laboratory, \\ Los Alamos, NM 87545, USA \\ *Correspondence to: zelenay@lanl.gov
}

This file includes:

Figs. S1 to S8

Tables $\mathrm{S} 1$ to $\mathrm{S} 4$

Examples of free energy calculations 


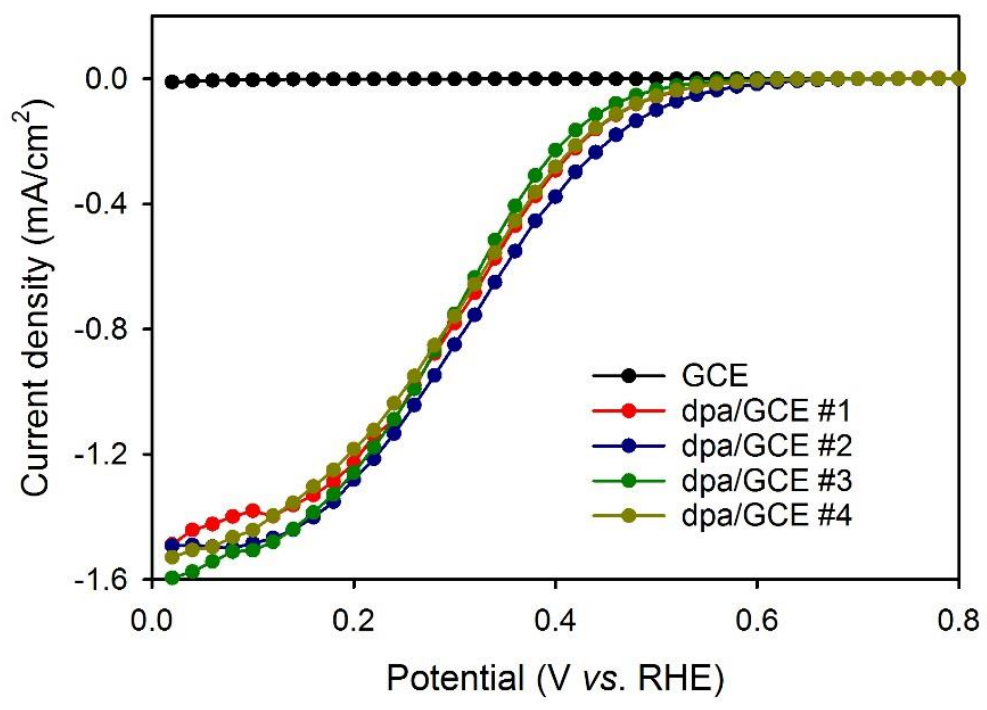

Figure S1. Multiple 2e-ORR polarization plots at dpa/GCE in $\mathrm{O}_{2}$-saturated $0.5 \mathrm{M} \mathrm{H}_{2} \mathrm{SO}_{4}$ at $25^{\circ} \mathrm{C}$, RDE rotation rate $900 \mathrm{rpm}, 20 \mathrm{mV}$ potential step, potential hold time $20 \mathrm{~s}$. Plot \#1 was recorded using Certified ACS Plus $\mathrm{H}_{2} \mathrm{SO}_{4}$, Fisher Chemical (lot\# 153943, each type of metal impurity less than 0.1 ppm). Plots \#2-4 were recorded using Optima ${ }^{\circledR} \mathrm{H}_{2} \mathrm{SO}_{4}$, Fisher Chemical (lot\# 3217051, most metal impurities less than 0.01 to $1 \mathrm{ppt}$ ). The Certificates of Analysis for the $\mathrm{H}_{2} \mathrm{SO}_{4}$ provided by the manufacturer are attached as additional files.

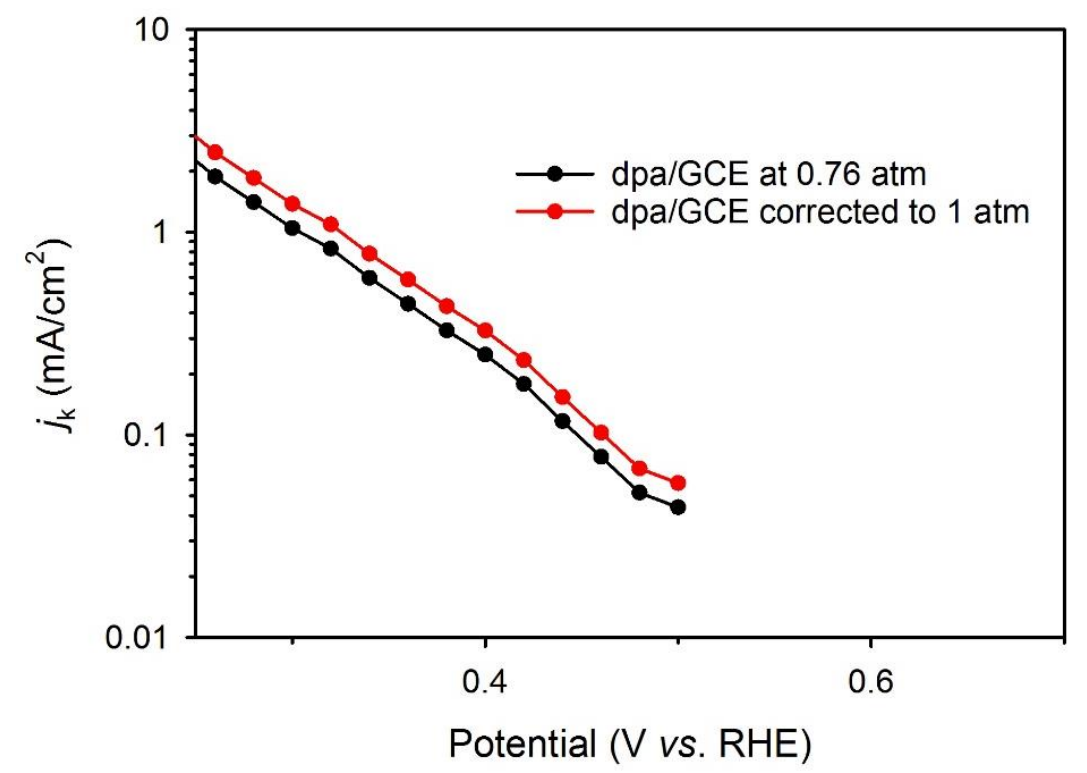

Figure S2. Kinetic current density for $\mathrm{H}_{2} \mathrm{O}_{2}$ generation at dpa/GCE, without (black) and with the correction (red) for the lower atmospheric pressure at Los Alamos, New Mexico. 


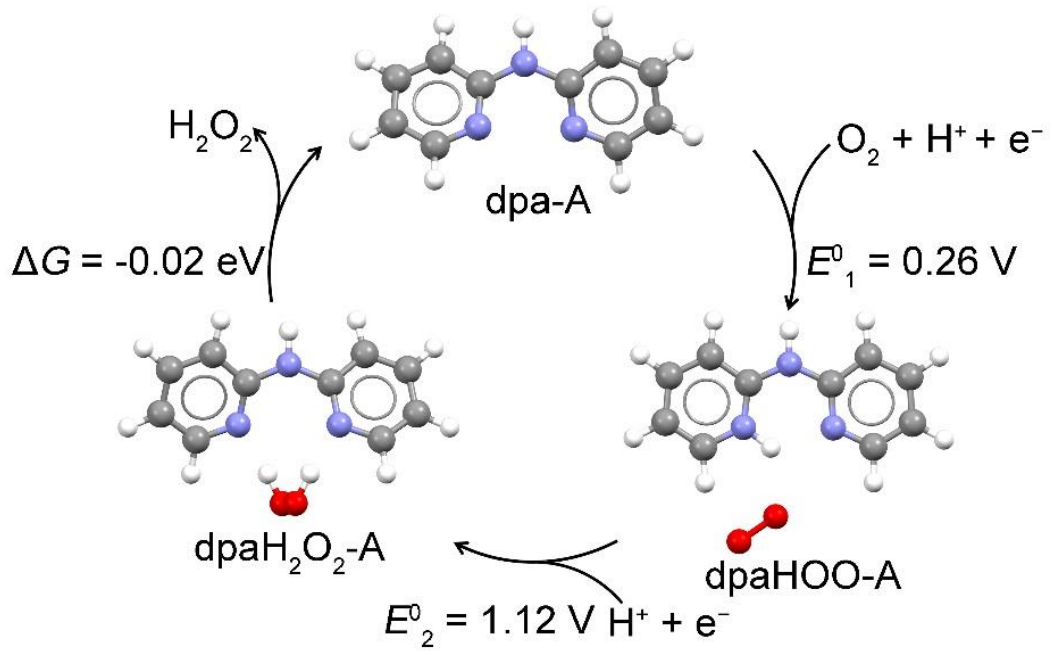

Figure S3. Catalytic cycle for 2e-ORR at dpa-A according to Mechanism I.

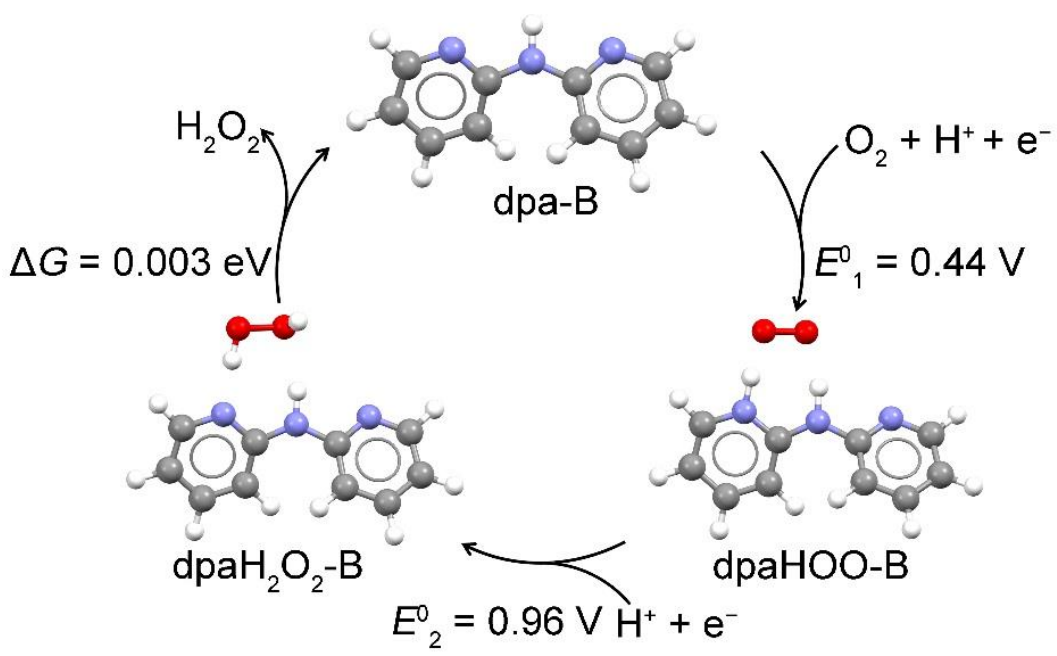

Figure S4. Catalytic cycle at dpa-B according to Mechanism I. 


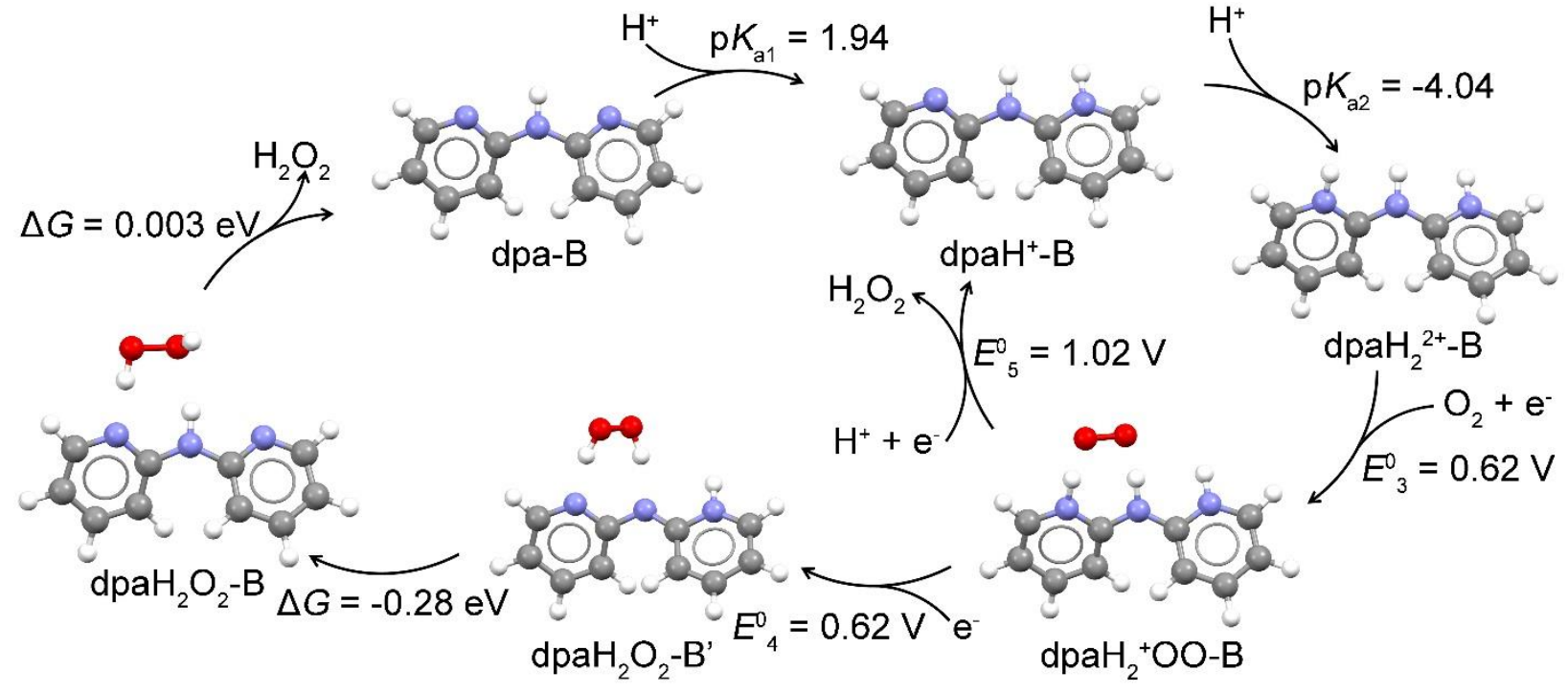

Figure S5. Catalytic cycle at dpa-B according to Mechanisms II and III.

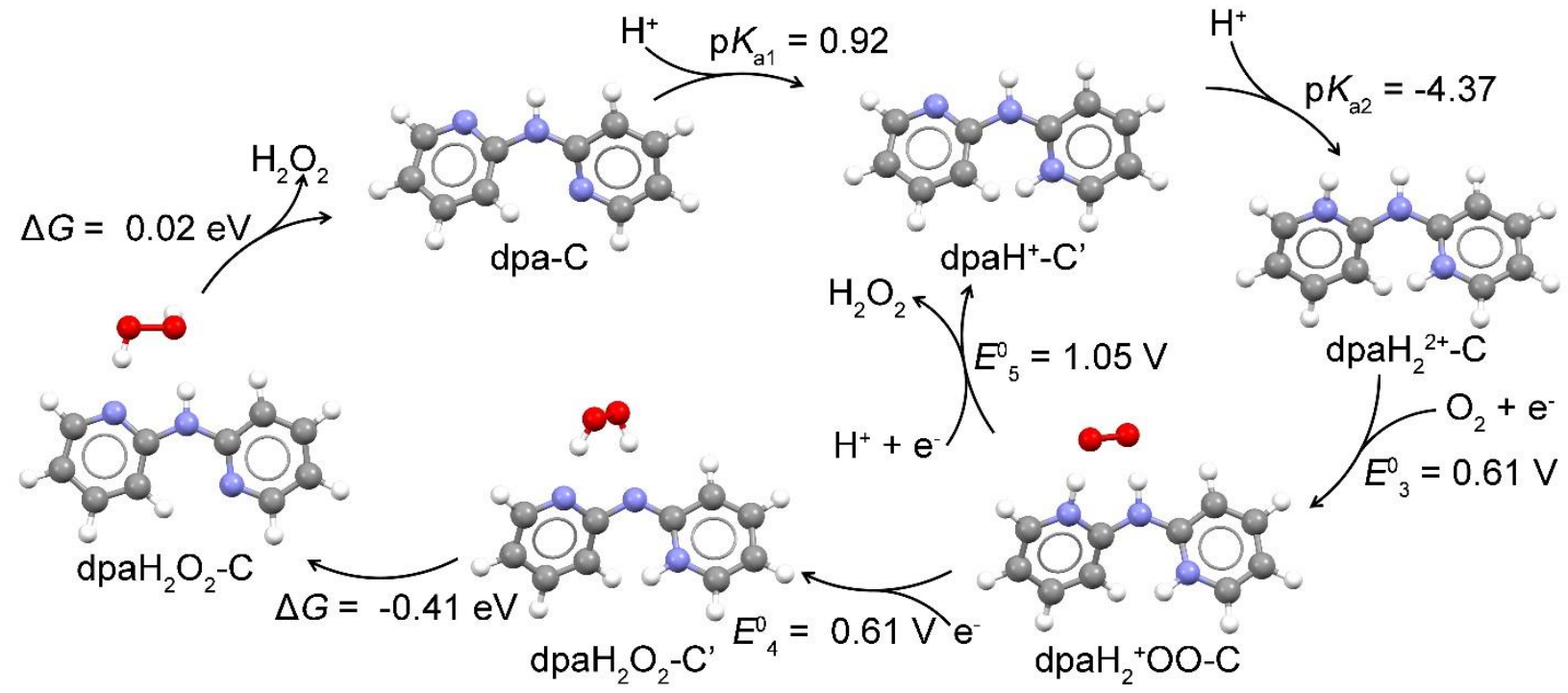

Figure S6. Catalytic cycle at dpa-C according to Mechanisms II and III. 

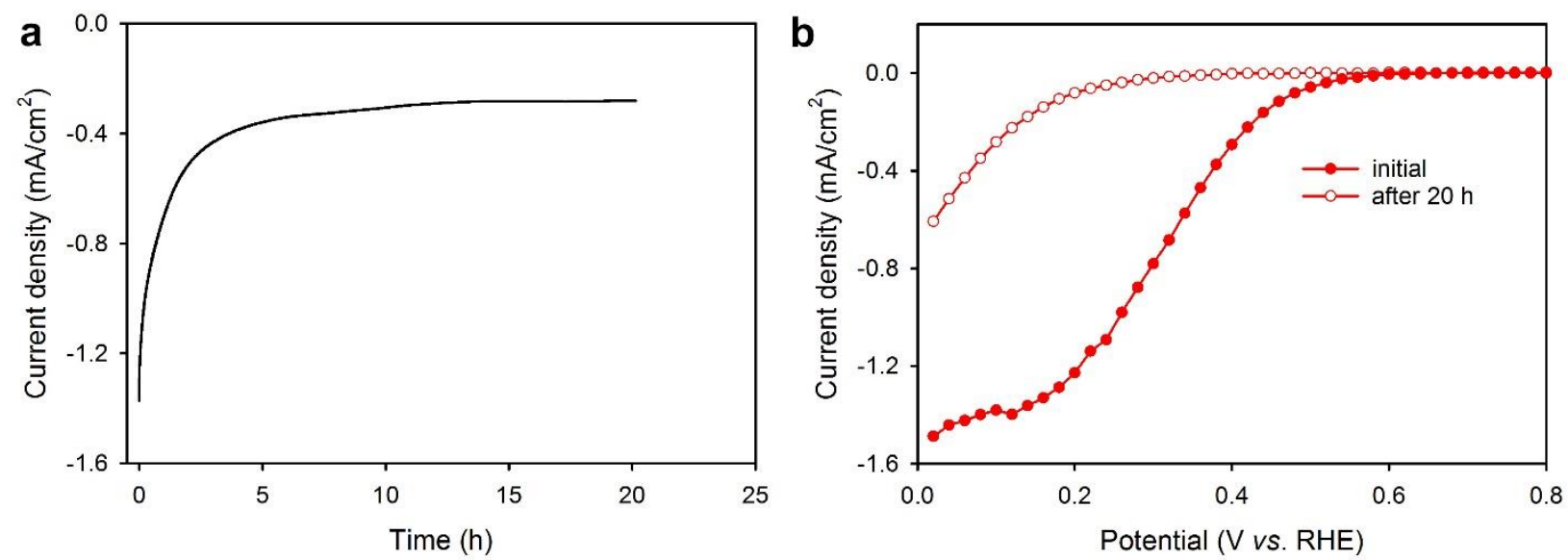

Figure S7. Durability of the dpa/GCE catalyst for $\mathrm{H}_{2} \mathrm{O}_{2}$ generation in $\mathrm{O}_{2}$-saturated $0.5 \mathrm{M} \mathrm{H}_{2} \mathrm{SO}_{4}$ at $25^{\circ} \mathrm{C}$. RDE rotation rate $900 \mathrm{rpm}$. (a) Current density $v s$. time curve at $0 \mathrm{~V} v s$. RHE. (b) 2eORR polarization plots measured before and after $20 \mathrm{~h}$ of a durability test ( $20 \mathrm{mV}$ steps, $20 \mathrm{~s} / \mathrm{step})$.

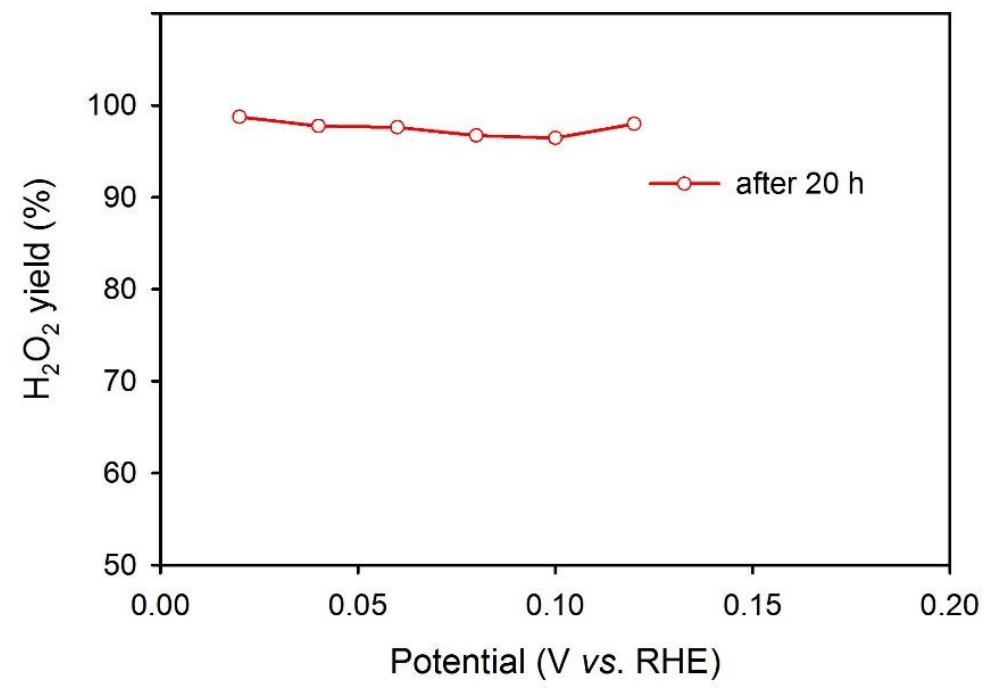

Figure S8. $\mathrm{H}_{2} \mathrm{O}_{2}$ yield at dpa/GCE after $20 \mathrm{~h}$ durability test. 
Table S1. ICP-MS analysis of as-received 2,2'-dipyridylamine.

\begin{tabular}{|c|c|c|c|}
\hline Element & Concentration (ppm) & Element & Concentration (ppm) \\
\hline $\mathrm{Li}$ & 0.663 & $\mathrm{Cd}$ & 0.000 \\
\hline $\mathrm{Be}$ & 0.000 & In & 0.000 \\
\hline $\mathrm{B}$ & 0.000 & Sn & 0.000 \\
\hline $\mathrm{Na}$ & 92.333 & $\mathrm{Sb}$ & 0.000 \\
\hline $\mathrm{Mg}$ & 26.195 & $\mathrm{Te}$ & 0.000 \\
\hline $\mathrm{Al}$ & 0.000 & I & 0.000 \\
\hline $\mathrm{Si}$ & 0.000 & Cs & 0.015 \\
\hline $\mathrm{P}$ & 53.701 & $\mathrm{Ba}$ & 0.568 \\
\hline $\mathrm{S}$ & 0.000 & $\mathrm{La}$ & 0.038 \\
\hline $\mathrm{K}$ & 65.031 & $\mathrm{Ce}$ & 0.000 \\
\hline $\mathrm{Ca}$ & 0.000 & $\operatorname{Pr}$ & 0.007 \\
\hline $\mathrm{Sc}$ & 0.000 & $\mathrm{Nd}$ & 0.000 \\
\hline $\mathrm{Ti}$ & 0.000 & $\mathrm{Sm}$ & 0.000 \\
\hline $\mathrm{V}$ & 0.000 & $\mathrm{Eu}$ & 0.000 \\
\hline $\mathrm{Cr}$ & 0.012 & $\mathrm{Gd}$ & 0.000 \\
\hline $\mathrm{Mn}$ & 1.810 & $\mathrm{~Tb}$ & 0.000 \\
\hline $\mathrm{Fe}$ & 0.000 & Dy & 0.000 \\
\hline Co & 0.091 & Ho & 0.003 \\
\hline $\mathrm{Ni}$ & 0.890 & $\mathrm{Er}$ & 0.000 \\
\hline $\mathrm{Cu}$ & 23.311 & $\mathrm{Tm}$ & 0.000 \\
\hline $\mathrm{Zn}$ & 14.019 & $\mathrm{Yb}$ & 0.000 \\
\hline $\mathrm{Ga}$ & 0.000 & $\mathrm{Lu}$ & 0.000 \\
\hline $\mathrm{Ge}$ & 0.000 & $\mathrm{Hf}$ & 0.000 \\
\hline As & 0.000 & $\mathrm{Ta}$ & 0.000 \\
\hline $\mathrm{Se}$ & 0.000 & $\mathrm{~W}$ & 0.000 \\
\hline $\mathrm{Br}$ & 0.000 & $\operatorname{Re}$ & 0.000 \\
\hline $\mathrm{Kr}$ & 0.000 & Os & 0.000 \\
\hline $\mathrm{Rb}$ & 0.121 & $\mathrm{Ir}$ & 0.000 \\
\hline $\mathrm{Sr}$ & 0.613 & $\mathrm{Pt}$ & 0.000 \\
\hline $\mathrm{Y}$ & 0.000 & $\mathrm{Au}$ & 0.000 \\
\hline $\mathrm{Zr}$ & 0.000 & $\mathrm{Hg}$ & 0.000 \\
\hline $\mathrm{Nb}$ & 0.013 & $\mathrm{Tl}$ & 0.000 \\
\hline Mo & 0.000 & $\mathrm{~Pb}$ & 2.510 \\
\hline $\mathrm{Ru}$ & 0.000 & $\mathrm{Bi}$ & 3.647 \\
\hline $\mathrm{Rh}$ & 0.006 & Th & 0.021 \\
\hline $\mathrm{Pd}$ & 0.000 & $\mathrm{U}$ & 0.158 \\
\hline $\mathrm{Ag}$ & 0.000 & & \\
\hline
\end{tabular}


Table S2. ICP-AAS analysis of glassy carbon SIGRADUR ${ }^{\circledR}$ provided by HochtemperaturWerkstoffe GmbH

\begin{tabular}{|c|c|}
\hline Element & Concentrations (ppm) \\
\hline $\mathrm{Al}$ & 0.27 \\
\hline $\mathrm{Ba}$ & 0.081 \\
\hline $\mathrm{Be}$ & 0.044 \\
\hline $\mathrm{Bi}$ & 0.050 \\
\hline $\mathrm{Ca}$ & 9.71 \\
\hline $\mathrm{Cd}$ & 0.044 \\
\hline $\mathrm{Co}$ & 0.089 \\
\hline $\mathrm{Cr}$ & 0.091 \\
\hline $\mathrm{Cu}$ & 0.030 \\
\hline $\mathrm{Fe}$ & 0.92 \\
\hline $\mathrm{K}$ & 0.34 \\
\hline $\mathrm{Mg}$ & 0.052 \\
\hline $\mathrm{Mn}$ & 0.041 \\
\hline $\mathrm{Mo}$ & 0.044 \\
\hline $\mathrm{Na}$ & 0.78 \\
\hline $\mathrm{Ni}$ & 0.12 \\
\hline $\mathrm{Sn}$ & 0.44 \\
\hline $\mathrm{Si}$ & 13.86 \\
\hline $\mathrm{Sr}$ & 0.061 \\
\hline $\mathrm{Ti}$ & 0.096 \\
\hline $\mathrm{V}$ & 0.044 \\
\hline $\mathrm{W}$ & 0.089 \\
\hline $\mathrm{Zn}$ & 0.044 \\
\hline $\mathrm{Zr}$ & 0.062 \\
\hline
\end{tabular}


Table S3. Gibbs free energy of dpa and reaction intermediates from DFT calculation under standard conditions (298.15 K, 1 atm, 1 M concentration in water)

\begin{tabular}{|c|c|}
\hline Structure & $\begin{array}{l}\text { B3LYP/6-31++G(d,p)/IEFPCM-water free energy } \\
\text { (Hartree) }\end{array}$ \\
\hline dpa-A & -550.647003 \\
\hline dpa-B & -550.650825 \\
\hline dpa-C & -550.653268 \\
\hline $\mathrm{dpaH}{ }^{+}-\mathrm{A}$ & -551.094832 \\
\hline $\mathrm{dpaH}^{+}-\mathrm{B}$ & -551.081396 \\
\hline $\mathrm{dpaH}^{+}-\mathrm{C}$ & -551.083816 \\
\hline $\mathrm{dpaH}^{+}-\mathrm{C}^{\prime}$ & -551.081622 \\
\hline dpaHOO-A & -701.572811 \\
\hline dpaHOO-B & -701.583410 \\
\hline dpaHOO-C & -701.586923 \\
\hline dpaHOO-C' & -701.581574 \\
\hline $\mathrm{dpaH}_{2} \mathrm{O}_{2}-\mathrm{A}$ & -702.203987 \\
\hline $\mathrm{dpaH}_{2} \mathrm{O}_{2}-\mathrm{B}$ & -702.208784 \\
\hline $\mathrm{dpaH}_{2} \mathrm{O}_{2}-\mathrm{B}^{\prime}$ & -702.198524 \\
\hline $\mathrm{dpaH}_{2} \mathrm{O}_{2}-\mathrm{C}$ & -702.211920 \\
\hline $\mathrm{dpaH}_{2} \mathrm{O}_{2}-\mathrm{C}^{\prime}$ & -702.196765 \\
\hline $\mathrm{dpaH}_{2}{ }^{+} \mathrm{OO}-\mathrm{A}$ & -702.011930 \\
\hline $\mathrm{dpaH}_{2}{ }^{+} \mathrm{OO}-\mathrm{B}$ & -702.011760 \\
\hline $\mathrm{dpaH}_{2}^{+} \mathrm{OO}-\mathrm{C}$ & -702.010675 \\
\hline $\mathrm{dpaH}_{2}^{2+}-\mathrm{A}$ & -551.496073 \\
\hline $\mathrm{dpaH}_{2}{ }^{2+}-\mathrm{B}$ & -551.498974 \\
\hline $\mathrm{dpaH}_{2}{ }^{2+}-\mathrm{C}$ & -551.498483 \\
\hline
\end{tabular}

Table S4. Parameterized Gibbs free energies of reference states at 298.15K, 1 atm pressure.

\begin{tabular}{|c|c|}
\hline Reference states & Gibbs free energy (Hartree) \\
\hline $\mathrm{H}_{2}$ gas, 1 atm & -1.180339 \\
\hline solvated $\mathrm{H}^{+}(1 \mathrm{M}$, aqueous $)$ & -0.426352 \\
\hline free $\mathrm{e}^{-}$gas & -0.001383 \\
\hline $\mathrm{H}_{2} \mathrm{O}$ gas, 0.03128 atm & -76.433777 \\
\hline $\mathrm{H}_{2} \mathrm{O}$ (liquid) & -76.433777 \\
\hline $\mathrm{O}_{2}$ gas, 1 atm & -150.326067 \\
\hline $\mathrm{H}_{2} \mathrm{O}_{2}(1 \mathrm{M}$, aqueous $)$ & -151.557856 \\
\hline
\end{tabular}




\section{Calculations of the free energy change and standard potential for specific reactions}

$$
\begin{aligned}
& \Delta G_{\text {rxn }}\left(\text { dpa- } \mathrm{A}+\mathrm{H}^{+}+\mathrm{O}_{2}+\mathrm{e}^{-} \rightarrow \text { dpaHOO-A }\right)=-4.68 \mathrm{eV} ; E^{0}{ }_{1}=0.26 \mathrm{~V} \\
& \Delta G_{\text {rxn }}\left(\mathrm{dpaHOO}-\mathrm{A}+\mathrm{H}^{+}+\mathrm{e}^{-} \rightarrow \mathrm{dpaH}_{2} \mathrm{O}_{2}-\mathrm{A}\right)=-5.54 \mathrm{eV} ; E^{0}{ }_{2}=1.12 \mathrm{~V} \\
& \Delta G_{\text {rxn }}\left(\text { dpaH }_{2} \mathrm{O}_{2}-\mathrm{A} \rightarrow \text { dpa-A }+\mathrm{H}_{2} \mathrm{O}_{2}\right)=-0.024 \mathrm{eV} \\
& \Delta G_{\mathrm{rxn}}\left(\mathrm{dpa}-\mathrm{B}+\mathrm{H}^{+}+\mathrm{O}_{2}+\mathrm{e}^{-} \rightarrow \text { dpaHOO-B }\right)=-4.86 \mathrm{eV} ; E^{0}{ }_{1}=0.44 \mathrm{~V} \\
& \Delta G_{\text {rxn }}\left(\text { dpaHOO-B }+\mathrm{H}^{+}+\mathrm{e}^{-} \rightarrow \mathrm{dpaH}_{2} \mathrm{O}_{2}-\mathrm{B}\right)=-5.38 \mathrm{eV} ; E^{0}{ }_{2}=0.96 \mathrm{~V} \\
& \Delta G_{\mathrm{rxn}}\left(\mathrm{dpaH}_{2} \mathrm{O}_{2}-\mathrm{B} \rightarrow \text { dpa-B }+\mathrm{H}_{2} \mathrm{O}_{2}\right)=0.003 \mathrm{eV} \\
& \Delta G_{\text {rxn }}\left(\mathrm{dpa}-\mathrm{C}+\mathrm{H}^{+}+\mathrm{O}_{2}+\mathrm{e}^{-} \rightarrow \text { dpaHOO-C }\right)=-4.89 \mathrm{eV} ; E^{0}=0.47 \mathrm{~V} \\
& \Delta G_{\text {rxn }}\left(\text { dpaHOO-C }+\mathrm{H}^{+}+\mathrm{e}^{-} \rightarrow \mathrm{dpaH}_{2} \mathrm{O}_{2}-\mathrm{C}\right)=-5.37 \mathrm{eV} ; E^{0}=0.95 \mathrm{~V} \\
& \Delta G_{\text {rxn }}\left(\text { dpaH }_{2} \mathrm{O}_{2}-\mathrm{C} \rightarrow \text { dpa- } \mathrm{C}+\mathrm{H}_{2} \mathrm{O}_{2}\right)=0.022 \mathrm{eV} \\
& \Delta G_{\text {rxn }}\left(\mathrm{dpaH}_{2}{ }^{2+}-\mathrm{A}+\mathrm{O}_{2}+\mathrm{e}^{-} \rightarrow \mathrm{dpaH}_{2}{ }^{+} \mathrm{OO}-\mathrm{A}\right)=-5.13 \mathrm{eV} ; E^{0}{ }_{3}=0.71 \mathrm{~V} \\
& \Delta G_{\text {rxn }}\left(\mathrm{dpaH}_{2}{ }^{+} \mathrm{OO}-\mathrm{A}+\mathrm{e}^{-} \rightarrow \mathrm{dpaH}_{2} \mathrm{O}_{2}-\mathrm{A}\right)=-5.19 \mathrm{eV} ; E^{0}{ }_{4}=0.77 \mathrm{~V} \\
& \Delta G_{\text {rxn }}\left(\mathrm{dpaH}_{2}{ }^{+} \mathrm{OO}-\mathrm{A}+\mathrm{H}^{+}+\mathrm{e}^{-} \rightarrow \mathrm{dpaH}^{+}-\mathrm{A}+\mathrm{H}_{2} \mathrm{O}_{2}\right)=-5.80 \mathrm{eV} ; E^{0}{ }_{5}=1.38 \mathrm{~V} \\
& \Delta G_{\text {rxn }}\left(\mathrm{dpaH}_{2}{ }^{2+}-\mathrm{B}+\mathrm{O}_{2}+\mathrm{e}^{-} \rightarrow \mathrm{dpaH}_{2}{ }^{+} \mathrm{OO}-\mathrm{B}\right)=-5.04 \mathrm{eV} ; \mathrm{E}^{0}=0.62 \mathrm{~V} \\
& \Delta G_{\text {rxn }}\left(\mathrm{dpaH}_{2}{ }^{+} \mathrm{OO}-\mathrm{B}+\mathrm{e}^{-} \rightarrow \mathrm{dpaH}_{2} \mathrm{O}_{2}-\mathrm{B}^{\prime}\right)=-5.04 \mathrm{eV} ; \mathrm{E}^{0}=0.62 \mathrm{~V} \\
& \Delta G_{\text {rxn }}\left(\mathrm{dpaH}_{2}{ }^{+} \mathrm{OO}-\mathrm{B}+\mathrm{H}^{+}+\mathrm{e}^{-} \rightarrow \mathrm{dpaH}^{+}-\mathrm{B}+\mathrm{H}_{2} \mathrm{O}_{2}\right)=-5.44 \mathrm{eV} ; \mathrm{E}^{0}=1.02 \mathrm{~V} \\
& \Delta G_{\text {rxn }}\left(\mathrm{dpaH}_{2} \mathrm{O}_{2}-\mathrm{B}^{\prime} \rightarrow \mathrm{dpaH}_{2} \mathrm{O}_{2}-\mathrm{B}\right)=-0.28 \mathrm{eV} \\
& \Delta G_{\text {rxn }}\left(\text { dpaH}_{2} \mathrm{O}_{2}-\mathrm{B} \rightarrow \text { dpa-B }+\mathrm{H}_{2} \mathrm{O}_{2}\right)=0.003 \mathrm{eV} \\
& \Delta G_{\text {rxn }}\left(\mathrm{dpaH}_{2}{ }^{2+}-\mathrm{C}+\mathrm{O}_{2}+\mathrm{e}^{-} \rightarrow \mathrm{dpaH}_{2}{ }^{+} \mathrm{OO}-\mathrm{C}\right)=-5.03 \mathrm{eV} ; \mathrm{E}^{0}=0.61 \mathrm{~V} \\
& \Delta G_{\text {rxn }}\left(\mathrm{dpaH}_{2}{ }^{+} \mathrm{OO}-\mathrm{C}+\mathrm{e}^{-} \rightarrow \mathrm{dpaH}_{2} \mathrm{O}_{2}-\mathrm{C}^{\prime}\right)=-5.03 \mathrm{eV} ; \mathrm{E}^{0}=0.61 \mathrm{~V} \\
& \Delta G_{\text {rxn }}\left(\mathrm{dpaH}_{2}{ }^{+} \mathrm{OO}-\mathrm{C}+\mathrm{H}^{+}+\mathrm{e}^{-} \rightarrow \mathrm{dpaH}^{+}-\mathrm{C}^{\prime}+\mathrm{H}_{2} \mathrm{O}_{2}\right)=-5.47 \mathrm{eV} ; \mathrm{E}^{0}=1.05 \mathrm{~V} \\
& \Delta G_{\text {rxn }}\left(\mathrm{dpaH}_{2} \mathrm{O}_{2}-\mathrm{C}^{\prime} \rightarrow \mathrm{dpaH}_{2} \mathrm{O}_{2}-\mathrm{C}\right)=-0.41 \mathrm{eV} \\
& \Delta G_{\text {rxn }}\left(\text { dpaH }_{2} \mathrm{O}_{2}-\mathrm{C} \rightarrow \text { dpa-C }+\mathrm{H}_{2} \mathrm{O}_{2}\right)=0.022 \mathrm{eV}
\end{aligned}
$$




\section{Free energy calculation using computational hydrogen electrode approach}

We used computational hydrogen electrode (CHE) approach to calculate the free energy level of intermediates in $\mathrm{CHE}$ scheme, denoted as $G_{\mathrm{CHE}}$. The following reference states were used in the calculations:

$G_{\mathrm{CHE}}\left(\mathrm{O}_{2}\right)=4 \times 1.23 \mathrm{eV}=4.92 \mathrm{eV}$

$G_{\mathrm{CHE}}\left(\mathrm{H}_{2} \mathrm{O}\right)=0 \mathrm{eV}$

$G_{\mathrm{CHE}}\left(\mathrm{H}_{2}\right)=0 \mathrm{eV}$

$G_{\mathrm{CHE}}\left(\mathrm{H}_{2} \mathrm{O}_{2}\right)=G_{\mathrm{CHE}}\left(\mathrm{O}_{2}\right)+G_{\mathrm{CHE}}\left(\mathrm{H}_{2}\right)-2 \times 0.7 \mathrm{eV}=3.52 \mathrm{eV}$

$G_{\mathrm{CHE}}(*)=0 \mathrm{eV}$

When electrode potential $U$ equals $0 \mathrm{~V}$, the free energy of $\mathrm{H}_{2}$ and $\left(\mathrm{H}^{+}+\mathrm{e}^{-}\right)$pair is at equilibrium.

$G_{\mathrm{CHE}, U=0}\left(\mathrm{H}^{+}+\mathrm{e}^{-}\right)=0.5 \times G_{\mathrm{CHE}}\left(\mathrm{H}_{2}\right)=0 \mathrm{eV}$

At potential $U$, the free energy the $\left(\mathrm{H}^{+}+\mathrm{e}^{-}\right)$pair can be calculated using the equation:

$G_{\mathrm{CHE}, U}\left(\mathrm{H}^{+}+\mathrm{e}^{-}\right)=0.5 \times G_{\mathrm{CHE}}\left(\mathrm{H}_{2}\right)-\mathrm{e} U$

The free energy level of $\left(*+\mathrm{O}_{2}+2\left(\mathrm{H}^{+}+\mathrm{e}^{-}\right)\right),\left(*+\mathrm{H}_{2} \mathrm{O}_{2}\right)$, and $\left(\mathrm{HOO}^{*}+\left(\mathrm{H}^{+}+\mathrm{e}^{-}\right)\right)$in CHE scheme at potential $U$ are calculated using the following equations:

$$
\begin{aligned}
& G_{\mathrm{CHE}, U}\left(*+\mathrm{O}_{2}+2\left(\mathrm{H}^{+}+\mathrm{e}^{-}\right)\right) \\
& =G_{\mathrm{CHE}}(*)+G_{\mathrm{CHE}}\left(\mathrm{O}_{2}\right)+2 \times G_{\mathrm{CHE}, U}\left(\mathrm{H}^{+}+\mathrm{e}^{-}\right) \\
& =G_{\mathrm{CHE}}(*)+G_{\mathrm{CHE}}\left(\mathrm{O}_{2}\right)+2 \times\left[0.5 \times G_{\mathrm{CHE}}\left(\mathrm{H}_{2}\right)-\mathrm{e} U\right] \\
& =0 \mathrm{eV}+4.92 \mathrm{eV}+2 \times[0.5 \times 0 \mathrm{eV}-\mathrm{e} U] \\
& =4.92 \mathrm{eV}-2 \mathrm{e} U
\end{aligned}
$$

$G_{\mathrm{CHE}, U}\left(*+\mathrm{H}_{2} \mathrm{O}_{2}\right)$

$=G_{\mathrm{CHE}}(*)+G_{\mathrm{CHE}}\left(\mathrm{H}_{2} \mathrm{O}_{2}\right)$

$=3.52 \mathrm{eV}$

$G_{\mathrm{CHE}, U}\left(\mathrm{HOO}^{*}+\left(\mathrm{H}^{+}+\mathrm{e}^{-}\right)\right)$

$=G_{\mathrm{CHE}}\left(\mathrm{HOO}^{*}\right)+G_{\mathrm{CHE}, U}\left(\mathrm{H}^{+}+\mathrm{e}^{-}\right)$

$=G_{\mathrm{CHE}}\left(\mathrm{HOO}^{*}\right)+\left[0.5 \times G_{\mathrm{CHE}}\left(\mathrm{H}_{2}\right)-\mathrm{e} U\right]$

$=G_{\mathrm{CHE}}\left(\mathrm{HOO}^{*}\right)-\mathrm{e} U$ 


$$
\begin{aligned}
& \Delta G\left(\mathrm{H}^{+}+\mathrm{e}^{-}+\mathrm{O}_{2}+* \rightarrow \mathrm{HOO}^{*}\right) \\
& =G_{\mathrm{CHE}}\left(\mathrm{HOO}^{*}\right)-G_{\mathrm{CHE}}\left(\mathrm{O}_{2}\right)-G_{\mathrm{CHE}}(*)-G_{\mathrm{CHE}, U}\left(\mathrm{H}^{+}+\mathrm{e}^{-}\right) \\
& =G_{\mathrm{CHE}}\left(\mathrm{HOO}^{*}\right)-G_{\mathrm{CHE}}\left(\mathrm{O}_{2}\right)-G_{\mathrm{CHE}}(*)-\left[0.5 \times G_{\mathrm{CHE}}\left(\mathrm{H}_{2}\right)-\mathrm{e} U\right] \\
& =G\left(\mathrm{HOO}^{*}\right)-G\left(\mathrm{O}_{2}\right)-G(*)-\left[0.5 \times G\left(\mathrm{H}_{2}\right)-\mathrm{e} U\right] \\
& G_{\mathrm{CHE}}\left(\mathrm{HOO}^{*}\right) \\
& =G\left(\mathrm{HOO}^{*}\right)-G\left(\mathrm{O}_{2}\right)-G(*)-\left[0.5 \times G\left(\mathrm{H}_{2}\right)-\mathrm{e} U\right]+G_{\mathrm{CHE}}\left(\mathrm{O}_{2}\right)+G_{\mathrm{CHE}}(*)+\left[0.5 \times G_{\mathrm{CHE}}\left(\mathrm{H}_{2}\right)-\mathrm{e} U\right] \\
& =G\left(\mathrm{HOO}^{*}\right)-G\left(\mathrm{O}_{2}\right)-G(*)-\left[0.5 \times G\left(\mathrm{H}_{2}\right)-\mathrm{e} U\right]+4.92 \mathrm{eV}+0 \mathrm{eV}+[0.5 \times 0 \mathrm{eV}-\mathrm{e} U] \\
& =G\left(\mathrm{HOO}^{*}\right)-G\left(\mathrm{O}_{2}\right)-G(*)-0.5 \times G\left(\mathrm{H}_{2}\right)+4.92 \mathrm{eV}
\end{aligned}
$$

The free energy of $\mathrm{HOO}^{*}$ in $\mathrm{CHE}$ scheme, $\Delta G_{\mathrm{HOO}} *$, or $G_{\mathrm{CHE}}(\mathrm{dpaHOO})$ can be calculated as: $G_{\mathrm{CHE}}(\mathrm{dpaHOO}-\mathrm{A})=G(\mathrm{dpaHOO}-\mathrm{A})-G\left(\mathrm{O}_{2}\right)-G(\mathrm{dpa}-\mathrm{A})-0.5 \times G\left(\mathrm{H}_{2}\right)+4.92 \mathrm{eV}=4.66 \mathrm{eV}$ $G_{\mathrm{CHE}}(\mathrm{dpaHOO}-\mathrm{B})=G(\mathrm{dpaHOO}-\mathrm{B})-G\left(\mathrm{O}_{2}\right)-G(\mathrm{dpa}-\mathrm{B})-0.5 \times G\left(\mathrm{H}_{2}\right)+4.92 \mathrm{eV}=4.48 \mathrm{eV}$ $G_{\mathrm{CHE}}(\mathrm{dpaHOO}-\mathrm{C})=G(\mathrm{dpaHOO}-\mathrm{C})-G\left(\mathrm{O}_{2}\right)-G(\mathrm{dpa}-\mathrm{C})-0.5 \times G\left(\mathrm{H}_{2}\right)+4.92 \mathrm{eV}=4.45 \mathrm{eV}$

At $U=0.70 \mathrm{~V}$

$G_{\mathrm{CHE}, U=0.7}\left(\mathrm{dpaHOO}-\mathrm{A}+\left(\mathrm{H}^{+}+\mathrm{e}^{-}\right)\right)=4.66 \mathrm{eV}-0.70 \mathrm{eV}=3.96 \mathrm{eV}$

$G_{\text {CHE, } U=0.7}\left(\right.$ dpaHOO-B $\left.+\left(\mathrm{H}^{+}+\mathrm{e}^{-}\right)\right)=4.48 \mathrm{eV}-0.70 \mathrm{eV}=3.78 \mathrm{eV}$

$G_{\text {CHE, } U=0.7}\left(\right.$ dpaHOO-C $\left.+\left(\mathrm{H}^{+}+\mathrm{e}^{-}\right)\right)=4.45 \mathrm{eV}-0.70 \mathrm{eV}=3.75 \mathrm{eV}$ 